\title{
The Topological Connectivity of the Independence Complex of Circular-Arc Graphs
}

\author{
Yousef Mathkal Abd Algani ${ }^{1,2}$ \\ ${ }^{1}$ Department of Mathematics, University of Haifa, Haifa. \\ ${ }^{2}$ Department of Mathematics, The Arab Academic College for Education, Haifa. Israel.
}

\section{Article Info}

Keywords: Topological Connectivity, Independence Complex, Circular-Arc Graphs.

2010 AMS: $97 K 30$

Received: 20 April 2019

Accepted: 25 September 2019

Available online: 26 December 2019

\section{Abstract}

Let us denoted the topological connectivity of a simplicial complex $C$ plus 2 by $\eta(C)$.

Let $\psi$ be a function from class of graphs to the set of positive integers together with $\infty$. Suppose $\psi$ satisfies the following properties:

1. $\psi\left(K_{0}\right)=0$.

2. For every graph $\mathrm{G}$ there exists an edge $e=(x, y)$ of $G$ such that

$$
\psi(G-e) \geq \psi(G)
$$

(where $G-e$ is obtained from $G$ by the removal of the edge $e$ ), and

$$
\psi(G-N(\{x, y\})) \geq \psi(G)-1
$$

then

$$
\eta(\mathscr{I}(G)) \geq \psi(G)
$$

(where $(G-N(\{x, y\}))$ is obtained from $G$ by the removal of all neighbors of $x$ and $y$ (including, of course, $x$ and $y$ themselves).

Let us denoted the maximal function satisfying the conditions above by $\psi_{0}$.

Berger [3] prove the following conjecture:

$$
\eta(\mathscr{I}(G))=\psi_{0}(G)
$$

for trees and completements of chordal graphs.

Kawamura [2] proved conjecture, for chordal graphs. Berger [3] proved Conjecture for trees and completements of chordal graphs. In this article I proved the following theorem: Let $G$ be a circular-arc graph $G$ if $\psi_{0}(G) \leq 2$ then $\eta(\mathscr{I}(G)) \leq 2$. Prior the attempt to verify the previously mentioned cases, we need a few preparations which will be discussed in the introduction.

\section{Introduction}

A non-empty collection $\mathrm{C}$ of sets is called a simplicial complex if it is hereditary, namely if $\sigma \in C$ and $\tau \subseteq \sigma$ imply $\tau \in C$. It is well known that every simplicial complex has a unique (up to homeomorphism) geometric realisation, namely an embedding in some space $\mathbb{R}^{n}$, in which every simplex $\sigma \in C$ is realized as a homeomorph of a simplex in $\mathbb{R}^{n}$.

The simplicial join of $X$ and $Y$. Denoted by $X * Y$ is the (geometric realization of) a simplicial complex defined by

$$
X * Y=\{\sigma \cup \tau \mid \sigma \in X \tau \in Y\}
$$


Although we use the same symbol $*$ to mean the join of graphs and the join of simplicial complex, this causes no confusion in the seqel. the join of simplicial complex $X$ with a singleton $v$ is the cone of $X$ with apex $v$, denoted $C_{v} X$. the suspension of $X$ the join of $S^{0}$ and $X$, is denoted by $\operatorname{susp} X$.

We shall identify a complex with its geometric realisation. The topological connectivity of a simplicial complex $C$ is the largest number $c$ such that for every number $k \leq c$, every embedding of the $k$-dimensional sphere $S^{k}$ is extendable to an embedding of the $k+1$-dimensional ball $B^{k+1}$ in $C$. The connectivity of a complex may be infinite. The connectivity of $C$, plus 2 ,is denoted by $\eta(C)$.the reason for this definition is that the addition of 2 makes the formulation of some results more elegant.

For a graph $G$ with the vertex set $V$, a subset $A$ of $V$ is said to be independent if no two distinct vertices of $A$ are adjacent. A subset $S$ of $V$ is said to be dominating in $G$ if every vertex $v \in V$ is adjacent to a vertex of $S$. The domination number $\gamma(G)$ is the minimum of the cardinality of dominating sets of $G: \gamma(G)=\min \{|S| \mid S$ is dominating in $G\}$.

For a graph $H$, we denote by $\mathscr{I}(H)$ the simplicial complex consisting of all independent sets of vertices in $H$. As before, let $V_{i}, i \leq m$ be a partition of the vertex set of a graph $G$. Given a vertex $v \in V(G)$, we write $i(v)$. for the index $i$ for which $v \in V_{i}$. For a set $\mathrm{Z}$ of vertices we write $I(Z)=\{i(z): z \in Z\}$. As usual, we denote the set $\{1, \ldots, m\}$ by $[m]$. Given a subset $I$ of $[m]$, we write $V_{I}$ for $\bigcup_{i \in I} V_{i}$.

Consider a graph $G$ with a partition $V_{1}, V_{2}, \ldots, V_{m}$ of its vertex set. A choice of one vertex from each set $V_{i}$ is called an independent system of representative (ISR) if the selected vertices are non-adjacent in $\mathrm{G}$.

Existence of ISR :

Theorem 1.1. If for all $I \subseteq[m]$

$$
\eta\left(\mathscr{I}\left(G\left[V_{I}\right]\right)\right) \geq|I|
$$

then the partition $\left(V_{i}\right)(i \leq m)$ of $V(G)$ has an ISR.

To exemplify these notions and the above theorem, consider a bipartite graph $G$, whose sides are the two parts $V_{1}$ and $V_{2}$ of the given partition. In this case there exists an ISR if and only if the graph is not complete bipartite. But not being complete bipartite means the existence if a connection in $\mathscr{I}(G)$ between the two simplices $V_{1}$ and $V_{2}$ of $\mathscr{I}(G)$. Thus, not being complete bipartite is tantamount to $\mathscr{I}(G)$ being connected, which, in the above terminology, means being 0-connected, which means that $\eta(\mathscr{I}(G)) \geq 2$. Thus is this example, the condition of the theorem is not only sufficient, but also necessary (Enough to inseart one arc between tow simplices to be connected).

Theorem 1.2. Let $\psi$ be a function from class of graphs to the set of positive integers together with $\infty$. Suppose $\psi$ satisfies the following properties:

1. $\psi\left(K_{0}\right)=0$.

2. For every graph $G$ there exists an edge $e=(x, y)$ of $G$ such that

$$
\psi(G-e) \geq \psi(G)
$$

(where $G-e$ is obtained from $G$ by the removal of the edge $e$ ), and

$$
\psi(G-N(\{x, y\})) \geq \psi(G)-1
$$

then

$$
\eta(\mathscr{I}(G)) \geq \psi(G)
$$

(where $(G-N(\{x, y\}))$ is obtained from $G$ by the removal of all neighbors of $x$ and $y$ (including, of course, $x$ and $y$ themselves).

In fact, there is a maximal function $\psi_{0}$ satisfying the conditions of the theorem. It is best described in terms of a game between two players, (I) and (II). Player (I) want to maximize the function $\psi$ in the theorem(and hence prove that $\psi$ is large), while player(II) wants to minimize $\psi$. Player (I) selects an edge $e=(x, y)$ in the graph given at the present stage of the game. Player (II) chooses between two possibilities: he or she either (1) deletes $e$ from the graph, or else (2) deletes all neighbors of $x$ and $y$ (including, of course, $x$ and $y$ themselves). the game ends when either there remains an isolated vertex, in which case $\psi$ is defined as $\infty$, or there are no remaining vertices, in which case $\psi$ is defined as the number of moves of player (II) of type (2).

We define $\psi_{0}(G)$ as the maximal value of $\psi(G)$ player (I) can achieve in the game. Theorem 1.2 then states that $\eta(\mathscr{I}(G)) \geq \psi(G)$. Aharoni, Berger and Ziv [1] suggested the following conjecture:

\section{Conjecture 1.3.}

$$
\eta(\mathscr{I}(G))=\psi_{0}(G)
$$

Kawamura [2] proved the conjecture for chordal graphs. Now we define a chordal graph: A graph is chordal if each of its cycles of four or more nodes has a chord, which is an edge joining two nodes that are not adjacent in the cycle. Chordal graphs are know to be perfect graphs. They are sometimes also called triangulated graphs.

The chordal graphs are the intersection graphs of subtrees of a tree A classical theorem of Dirac [4] states that For each chordal graph $G$, there exists a vertex, called a simplicial vertex, such that $N(v)$ is a complete graph.

In this article I proved the obove conjecture for Circular-arc graphs. The circular-arc graphs, defined as the intersection graphs of a set of arcs on the circle. Such a graph has one vertex for each arc in the set, and an edge between every pair of vertices corresponding to arcs that intersect. 
Formally, let

$$
I_{1}, I_{2} \ldots, I_{n} \subseteq S^{1}
$$

be a set of arcs. Then the corresponding circular-arc graph is $G=(V, E)$, where

$$
V=\left\{I_{1}, I_{2} \ldots, I_{n}\right\}
$$

and

$$
\left\{I_{\alpha}, I_{\beta}\right\} \in E \Leftrightarrow I_{\alpha} \bigcap I_{\beta} \neq \emptyset
$$

A family of arcs that corresponds to $G$ is called an arc model of $G$. In [3] the following was proved:

Theorem 1.4. Let $G$ be a minimal counterexample for conjecture 1.3. Then $G$ has no vertex of degree 1 .

Corollary 1.5. Conjecture 1.3 holds for every tree.

The following was also proved in [3]

Theorem 1.6. If $G$ is a connected chordal graph then $\psi_{0}(\bar{G})=\infty$.

Theorems 1.6 and Corollary 1.5 of Berger prove Conjecture 1.3 for trees and completements of chordal graphs. Kawamura [2] proved it for chordal graphs.

Theorem 1.7. Let $G$ be a chordal graph. then $\mathscr{I}(G)$ is either contractible or is homotopy equivialent to the wedge of finitely many spheres $\bigvee S^{k_{t}}$. where $k_{t} \geq \gamma(G)-1$ for each $k_{t}$.

Conversely, all finite wedges of phares appear as homotopy types of independence complex of chordal graphs.

thus

Observation 1.8. (1) Let $L$ be a simplicial complex and let $k_{1}, \ldots, k_{r}$ be subcomplexes of $L$ (repetitions allowed). Take mutually distinct points $u_{1}, \ldots, u_{r}$ and $v$ with $\left\{u_{1}, \ldots, u_{r}, v\right\} \cup L=\phi$. then the union

$$
X=C_{v} L \cup \bigcup_{i=1}^{r} C_{u_{i}} K_{i}
$$

Subject to the conditions

$$
\begin{aligned}
C_{u_{i}} K_{i} \cap C_{v} L & =K_{i} \text { and } \\
C_{u_{i}} K_{i} \cap C_{u_{j}} L & =K_{i} \cap K_{j}(i, j=1, \ldots r, i \neq j) .
\end{aligned}
$$

is homotopy equivalent to $\bigvee_{i=1}^{r} \operatorname{susp}_{i}$.

(2)The suspension susp $X$ is contractible for each contractible complex $X$.

(3) $\operatorname{susp}\left(\vee S^{k_{r}}\right) \simeq \vee S^{k_{r}+1}$.

The following lemma provides us with a clear view on the structure of independence complexes of chordal graphs. For a vertex $v$ of a graph $G$, let $I_{v}(G)$ be the subcomplex generated by the independent sets containing $v$ :

$$
I_{v}(G)=\{A \mid \text { there exists a simplex } B \in I(G) \text { such that } v \in B \text { and } A \subset B\} .
$$

Lemma 1.9. Let $G$ be a graph and let $v$ be a simplicial vertex of $G$. Enumerate $N(v)$ as $N(v)=\left\{w_{1}, \ldots, w_{r}\right\}$. then we have the following.

$$
I(G) \simeq \bigvee \operatorname{suspI}\left(G-N\left[w_{i}\right]\right) .
$$

For each chordal garph $G$, we define $\eta(G) \in\{0,1, \ldots, \infty\}$ as follows: if $I(G)$ is contractible, then let $\eta(G)=\infty$ and, if $I(G)$ is homotopy equivalent to the wedge $\bigvee S^{i_{r}}$, then let $\eta(G)=$ mini $_{r}$, the minimum dimension of the associated spheres. under this notation theorem 1.7 and lemmaa1.9 yield the following corollary.

Corollary 1.10. Let $G$ be a chordal graph.

(1) We have an inequality $\eta(G) \geq \gamma(G)-1$. (2) Let $v$ be a simplicial vertex of $G$ and enumerate $N(v)$ as $N(v)=\left\{w_{1}, \ldots, w_{r}\right\}$. then we have $\eta(G)=\min \left\{\eta\left(g-n\left[w_{i}\right]\right) \mid i=1, \ldots, r\right\}+1$. here we make a convention that $\min \{\infty, \ldots, \infty\}+1=\infty$.

For the path $P_{n}$ with $n$ edges, $I\left(P_{n}\right)$ is contractible if $n \equiv 0(\bmod 3)$ and is homotopy equivalent to the sphere of dimension $\left\lfloor\frac{n}{3}\right\rfloor$. hence we have

$$
\eta\left(P_{n}\right)=\left\{\begin{array}{ccc}
\infty & \text { if } & n \equiv 0(\bmod 3) \\
\left\lfloor\frac{n}{3}\right\rfloor & \text { otherwise }
\end{array}\right.
$$

It shoud be noted thatKazuhiro Kawwamura [2] was proved above for chordal graphes ( Theorem 1.7, Observation 1.8, Lemma 1.9 and Corollary 1.10).

\section{New Result}

In this article I proved the following theorem, about circular-arc graphes.

Theorem 2.1. Let $G$ be a circular-arc graph, if exist that $\psi_{0}(G) \leq 2$ then $\eta(\mathscr{I}(G)) \leq 2$. 


\section{Some simple graphs}

\subsection{Path Grap, $P_{n}$}

Definition 3.1. In graph theory, Graph $(V, E)$ with $n+1$ vertices, which is a simple path is called path graph and is denoted $P_{n}$. Formally, a set $V$ of vertices $V=\{1,2, \ldots, n+1\}$, and a set $E$ of edges $E=\{\{i, i+1\} \mid i=1,2, \ldots, n\}$.

(see Fig. 3.1).

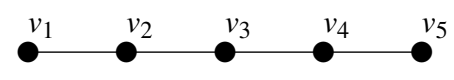

Fig: 3.1

Before we begin we want to define the concept of retraction:

Definition 3.2. Let $X$ be a topological space, let $A \subseteq X$, and let $i: A \hookrightarrow X$ be the inclusion map. A continuous map $f: X \rightarrow A$ is called a retraction if

$$
\left.f\right|_{A}=i d_{A}
$$

Correspondingly, $A$ is called a retract, a deformation retraction of $X$.

In the first stage we will explain the previously mention corollary 1.10: , but will begin by adding a chart which summarizes the main points regarding the corollary 1.10

$$
\eta\left(P_{n}\right)=\psi_{0}\left(P_{n}\right)=\left\{\begin{array}{ccc}
\infty & \text { if } & n \equiv 0(\bmod 3) \\
\left\lfloor\frac{n}{3}\right\rfloor & \text { otherwise }
\end{array}\right.
$$

\begin{tabular}{|c|c|c|c|c|}
\hline & Figure $\left(P_{n}\right)$ & $\mathscr{I}\left(P_{n}\right)$ & Figure $\mathscr{I}\left(P_{n}\right)$ & $\eta\left(P_{n}\right)$ \\
\hline$P_{1}$ & $\mathrm{P}^{v_{2}}$ & The independent complex disconnected & $0^{v_{2}}$ & $\eta\left(P_{1}\right)=1$ \\
\hline$P_{2}$ & & The independent complex disconnected & $0^{v_{1}}$ & $\eta\left(P_{2}\right)=1$ \\
\hline$P_{3}$ & $v_{1}$ & The independent complex is contractible. & $\overbrace{}^{v_{3}}$ & $\eta\left(P_{3}\right)=\infty$ \\
\hline$P_{4}$ & $0^{v_{1}} \overbrace{}^{v_{2}}$ & Now we can contractible $v_{3}$ into $v_{4}$ & (see Fig 1) & $\eta\left(P_{4}\right)=2$ \\
\hline
\end{tabular}

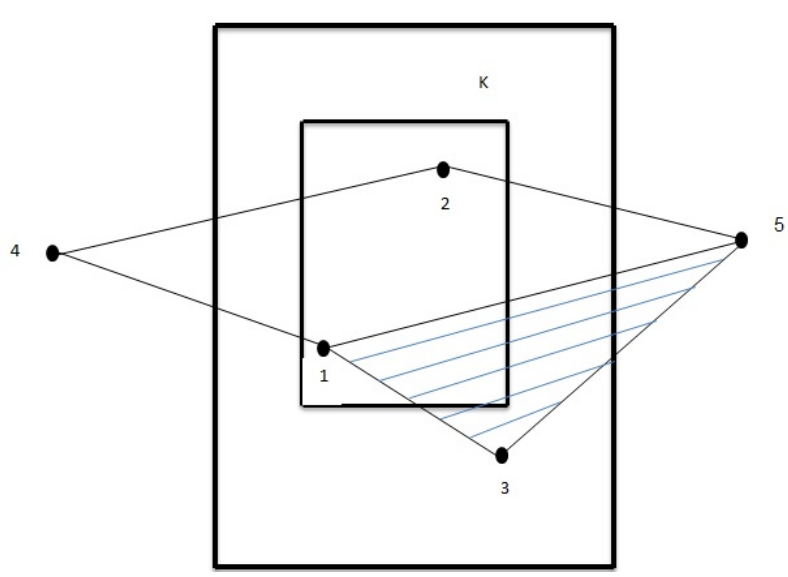

Figure 1: The independent complex of $P_{5}$.

Let's start from $P_{1}$ and $P_{2}$, the independent complex is not connected, then:

$$
\eta\left(P_{1}\right)=\eta\left(P_{2}\right)=1 \text {. }
$$


In the case $P_{3}$ the $\mathscr{I}\left(P_{3}\right)$ is contractible, then $\eta\left(P_{3}\right)=\infty$.

In the case $P_{4}$, let us denote the vertecies $v_{1}, v_{2} \ldots v_{5}$. In the $\mathscr{I}\left(P_{4}\right)$ (See Fig 1), let $L$ be a set that contains $\left\{v_{1}, v_{2}, v_{3}\right\}$, denote by $K$ the set of vertecies which are connected to $v_{4}$, and $v_{5}$ connected to the elements in $L$. Now we can contractible $v_{3}$ into $v_{4}$, ( and this does not change homotopy ).

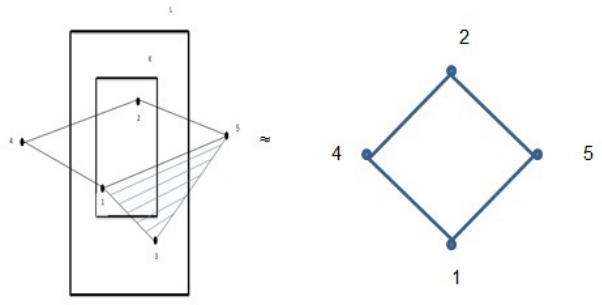

Figure 2: contractibtion $v_{3}$ into $v_{4}$.

then

$$
\eta\left(\operatorname{susp}\left(\mathscr{I}\left(P_{4}\right)\right)\right)=2 .
$$

(See Fig. 2 ).

In the case of $P_{7}$, we denote the vertecies $\left\{v_{1}, v_{2}, \ldots, v_{7}\right\}$. We will construct the independent complex as shown in the picture below ( See Fig.3), we notice that $N\left(v_{5}\right) \subseteq N\left(v_{7}\right)$, then we can contract $v_{5}$ into $v_{7}$.

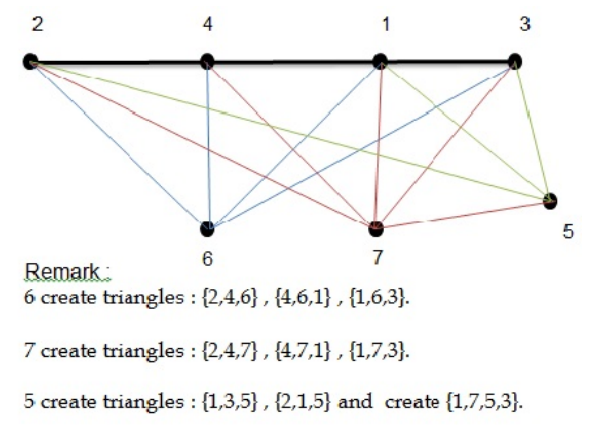

Figure 3: The independent complex of $P_{7}$.

After contraction, the result is Figure 4

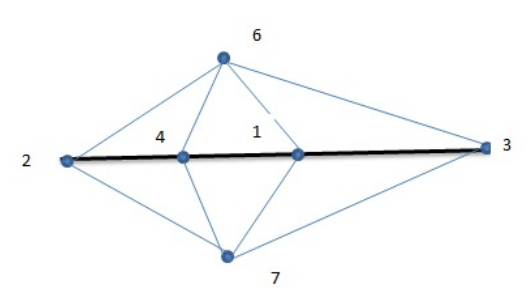

Figure 4: Contraction $v_{5}$ into $v_{7}$

In the case of $P_{10}$, we denote the vertecies $v_{1}, v_{2}, \ldots, v_{10}$. We construct the independent complex, we notice that $N\left(v_{8}\right) \subseteq N\left(v_{10}\right)$, then we can contract $v_{8}$ into $v_{10}$.

Now can prove the above case using induction, if we have two arcs $U \subseteq V$, (Fig 5), (If all the neighbors of $U$ contained in the neighbors of $V$ ) we can contract all independent sets in $U$ into $V$ and they remain independent.

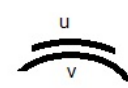




\subsection{Circle Grap, $C_{n}$}

Now we want to know the topology connectivity of a Circle Graph. At first I want to deine the Circle Graph.

Definition 3.3. A graph with $n$ vertexes which resembles a circle, is called a Circle Graph and is denoted by $C_{n}$. Formally, the graph set of vertexes is $V=\{0,1, \ldots, n-1\}$ and the arc's set is $E=\{\{i,(i+1) \bmod n\} \mid i=0,1, \ldots, n-1\}$.

We will start case $C_{5}$, and we will finde its topological connectivity:

3.2.1. $C_{5}$

In the first stage we built the independent complex of $C_{5}$ (Figure 6), and the $\mathscr{I}\left(C_{5}\right)$ is connected, but not simply connected then

$$
\eta\left(C_{5}\right)=2
$$

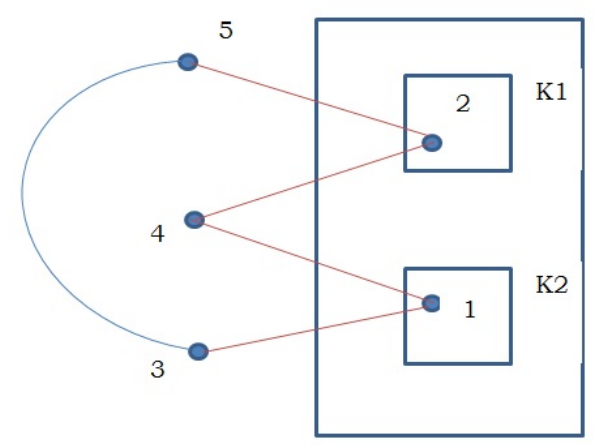

Figure 6: $\mathscr{I}\left(C_{5}\right)$

To get $\psi_{0}$ we want to remove an arc, Then the new graph is $P_{4}$. Then

$$
\eta\left(C_{5}\right)=\psi_{0}\left(C_{5}\right)=2
$$

3.2.2. $C_{6}$

At this stage we will show that

$$
\eta\left(C_{5}\right)=\psi_{0}\left(C_{5}\right)=2
$$

$C_{6}$ contains the vertexes $\left\{v_{1}, v_{2}, \ldots, v_{6}\right\}$. At the independent complex of $C_{6}$ (Figure 7), the $N\left(v_{4}\right)-\left(v_{1}\right.$ and $\left.v_{2}\right)$ denote by $K 1$, the $N\left(v_{6}\right)$ $\left(v_{2}\right.$ and $\left.v_{3}\right)$ denote by $K 2$, the $N\left(v_{5}\right)-\left(v_{1}, v_{2}\right.$ and $\left.v_{3}\right)$ denote by $L$.

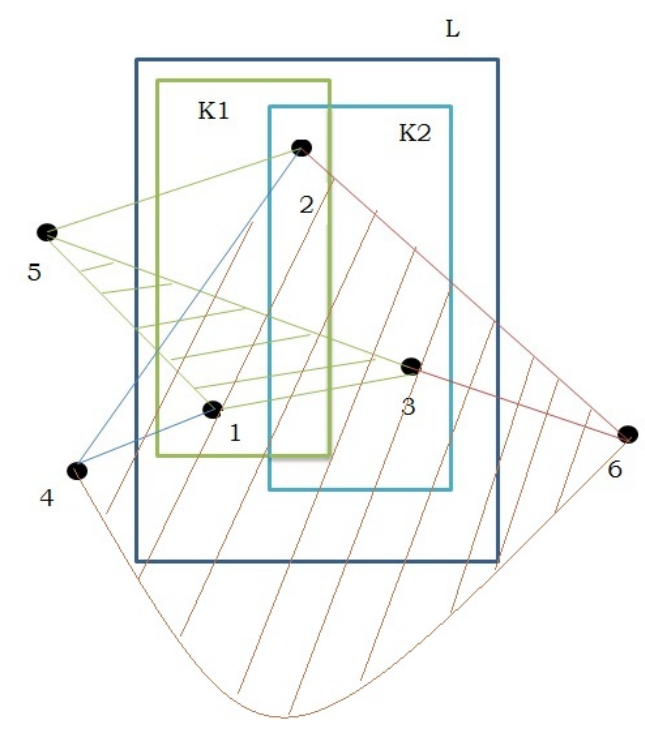

Figure 7: $\mathscr{I}\left(C_{6}\right)$ 
$\mathscr{I}\left(c_{6}\right) \cong \operatorname{susp}\left(\begin{array}{l}2 \\ \cdot \\ \cdot \\ 1\end{array}\right) \vee \operatorname{susp}\left(\begin{array}{l}2 \\ \cdot \\ \cdot \\ 3\end{array}\right)=$

For the same reason of the case $C_{5}: \psi_{0}=2$, then

$$
\psi_{0}\left(C_{6}\right)=\eta\left(C_{6}\right)=2
$$

3.2.3. $C_{8}$

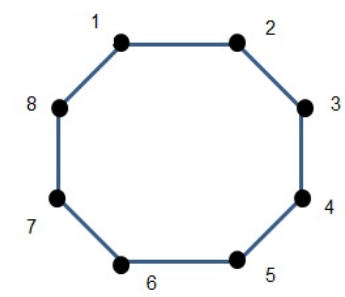

Figure 8: $\left(C_{8}\right)$

Constructing the independent complex of $C_{8}$ is not simple.

We denote the vertexes of $C_{8}$ by $\left\{v_{1}, v_{2}, \ldots, v_{8}\right\}$, see figure 8 .

We will construct the independent complex as follows:

We denote the neighbors of the vertex $v_{6}$ by $K 1$, and denote the neighbors of the vertex $v_{8}$ by $K 2$. The suspension of the form $F$ igure 9

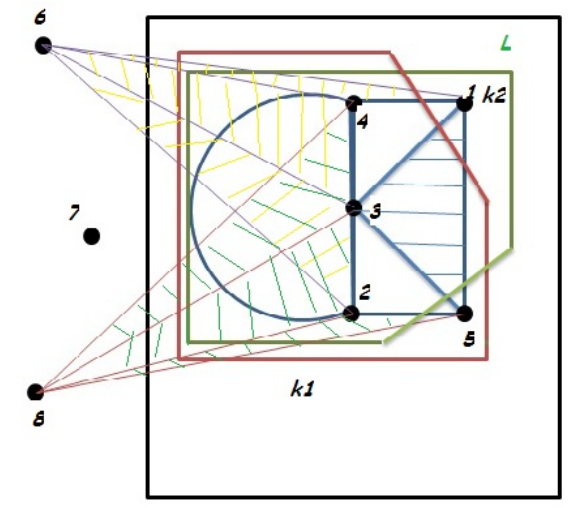

Figure 9: The first stage of constructing $\mathscr{I}\left(C_{8}\right)$

is homotope to:

$$
\mathscr{I}(\text { fig } 9) \cong \operatorname{susp}\left(\begin{array}{l}
2 \\
4 \\
1 \\
3
\end{array}\right) \vee \operatorname{susp}\left(\begin{array}{l}
4 \\
2 \\
5 \\
3
\end{array}\right)
$$

and we have the arc $\left\{v_{6}, v_{8}\right\}$. Ohterwise the independent complex is contactible and the topological connectivity will be $\infty$. Will complete construction of independent complex of $C_{8}$, and we will obtain the form seen in Fig.10. 


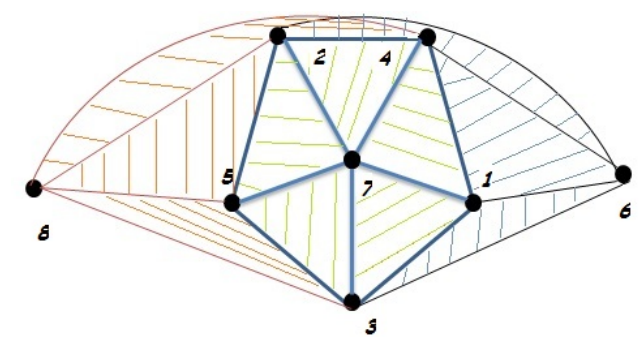

Figure 10: $\mathscr{I}\left(C_{8}\right)$

We have put two contractible independent complexes with anther contractible, that is the neighbors of $v_{7}$ (See Fig. 11).

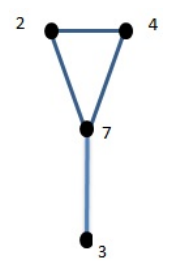

Figure 11: Contraction

We will contract the form Fig 11, to three vertexes $v_{2}, v_{4}$ and $v_{3} v_{2}^{v_{4}}$ and one arc $\left\{v_{2}, v_{4}\right\}$. We will contract $v_{2}^{v_{4}}$ denoted by $v_{24}$.

The independent complex of $C_{8}$ after the contraction, looks like Fig. 12, and that homotopy to $S^{2}$.

Then

$$
\eta\left(C_{8}\right)=3
$$

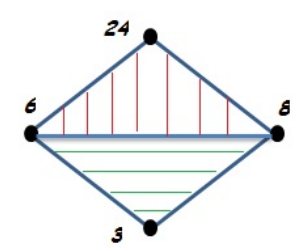

Figure 12: Contractible $\mathscr{I}\left(C_{8}\right)$

\subsubsection{General Case $C_{n}$}

Getting back to the last case $\left(C_{8}\right)$, and denoting $v_{8}$ by $n, v_{7}$ by $n-1$ and $v_{6}$ by $n-2$.

We have seen something else: if we ignore the relationship between $n$ and $n-2$, then we have obtained:

$$
\mathscr{I}\left(C_{n}\right) \cong \operatorname{Susp}\left(P_{n-5}\right) \bigvee \operatorname{Susp}\left(P_{n-5}\right) \bigvee \operatorname{Susp}\left(\operatorname{Susp}\left(P_{n-6}\right)\right) \text {. }
$$

and the value of $\eta\left(C_{n}\right)$ :

$$
\eta\left(C_{n}\right)=\min \left(\eta\left(P_{n-5}\right)+1, \eta\left(P_{n-6}\right)+2\right) .
$$

We have three cases:

1. If $n \equiv 0(\bmod 3)$

then $\eta\left(P_{n-6}\right)=\infty$, then the minimum is $\eta\left(P_{n-5}\right)+1=\left\lceil\frac{n-5}{3}\right\rceil+1$, and the result is:

$$
\left\lceil\frac{n-5}{3}\right\rceil+1=\frac{n}{3} \text {. }
$$

2. If $n \equiv 1(\bmod 3)$

then we get the minimum in $\eta\left(P_{n-5}\right)+1=\left\lceil\frac{n-5}{3}\right\rceil+1$ and the result is:

$$
\left\lceil\frac{n-5}{3}\right\rceil+1=\left\lfloor\frac{n}{3}\right\rfloor
$$


3. If $n \equiv 2(\bmod 3)$

then we get the minimum in $\eta\left(P_{n-6}\right)+2=\left\lceil\frac{n-6}{3}\right\rceil+2$ and the result is:

$$
\left\lceil\frac{n-6}{3}\right\rceil+2=\left\lceil\frac{n}{3}\right\rceil
$$

In the end, we have obtained the same result in the three cases:

$$
\begin{aligned}
& \frac{n}{3} . \\
& \eta\left(C_{n}\right)=\min \left(\eta\left(P_{n-5}\right)+1, \eta\left(P_{n-6}\right)+2\right)=\frac{n}{3} .
\end{aligned}
$$

\section{Proof of theorem 2.1}

Now we want to prove the theorem 2.1.

Let us denote the largest independent set in the cycle arc graph $G$ by $\alpha$. We need to prove that: if $\psi_{0} \leq 2$ then $\eta \leq 2$.

1. If $\alpha=1$ then $\psi_{0}=\eta=1$, because $G$ is a complete graph.

2. If $\alpha=2$, the independent complex of $G$ is $\bar{G},(\mathscr{I}(G)=\bar{G})$.

(a) If $\bar{G}$ not connected graph, then:

$$
\psi_{0}=\eta=1
$$

because $G$ contains a bipartite graph.

(b) If $\bar{G}$ is a tree [3] then:

$$
\psi_{0}=\eta=\infty .
$$

(c) If $\bar{G}$ is a connected graph but not a tree, then:

$$
\psi_{0}=\eta=2 \text {. }
$$

In order to see that $\eta=2$, note that there is a circle in $\bar{G}=\mathscr{I}(G)$ and we can not fill it. In order to see that $\psi_{0}=2$ it is enough to show that $\psi_{0} \geq 2$ and this can be achieved by using the strategy of always choosing an edge between two vertices of distance 2 in $\bar{G}$.

\section{If $\alpha=3$}

Recall that the collection of all simplices of $\mathscr{I}(G)$ of dimension less than or equal to $d$ is called the $d-$ skeleton of $\mathscr{I}(G)$, and is denoted by $\mathscr{I}(G)^{(d)}$. In particular, $\bar{G}$ is the $1-$ skeleton of $\mathscr{I}(G)$.

We now define a continuous function $f: \bar{G} \rightarrow S^{1}$ (where $\bar{G}$ is viewed as a topological space). The function maps each vertex to the middle of the appropriate segment, and maps each edge to an arc between the mid-segments according to the following rule. If there exists a third segment disjoint from both segments, then the arc goes through this third segment (see fig 13).

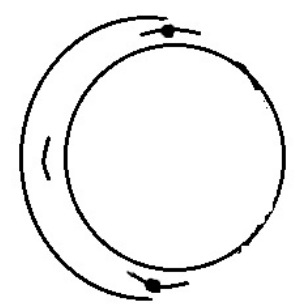

Figure 13: mapping vertics to mid-segment, and edges between mid-segment through the third segment.

The image of the triangle in $\bar{G}$ is arounded $S^{1}$ exactly twice.

Now the image of every triangle in $\bar{G}$ goes around $S^{1}$ an even number of times, and therefore it is impossible to fill a cycle in $\bar{G}$ that goes around $S^{1}$ an odd number of times.

4. $\alpha \geq 4$

Let $v_{1}, v_{2}, v_{3}, v_{4}$ be independent vertices.

Given sphare, and a segment corresponding to $v_{1}, v_{1}$ that does not contain any other segmet, but can stand out from side. We will review cutoff $v_{1}$ from all outstandingIt can stick out from the left side and the right side, (see fig.14). 


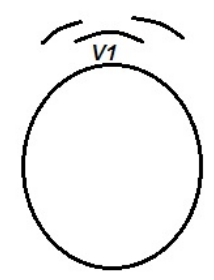

Figure 14: We will review cutoff $v_{1}$ from all aspects.

In the case of containment we remove the arc between the two segments (see fig.15).

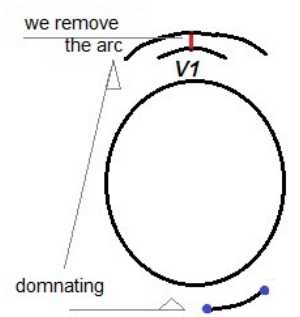

Figure 15: we remove the arc between the two segments.

For example, the first player chooses an arc, the second player removes all its neighbors, then: remains $\psi_{0}=1$, and the new graph is chordal.

In the case of a sticking out from the left side or the right side:

We begin from the segment in the least standing out to the longest, which we cannot remove, this vertex we denote by $x$.(See fig.16).

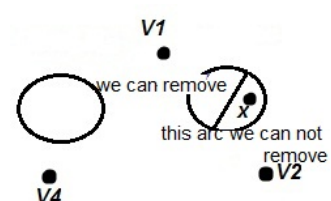

$\dot{v}$

Figure 16: The longest arc, we can not remove.

If we remove $\left\{v_{1}, x\right\}$ and all its neighbors, then a dominating vertex will exist, which we denote by $y$.

We claim that the vertices $x$ and $y$ is dominating set.

If $x$ is connected to $v_{1}, v_{2}$ and $v_{3}$, then we can remove $\left\{x, v_{2}\right\}$. We can not disconnect $y$ from $v_{3}$, because, the result would be bipartite graph, (we have a dominating vertex), (see fig.17).

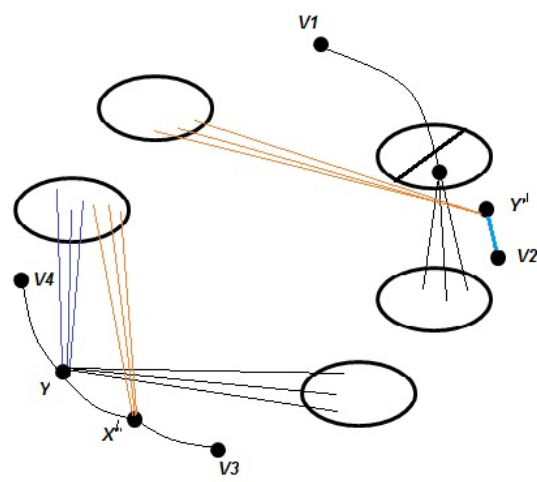

Figure 17: The structure of the graph. 


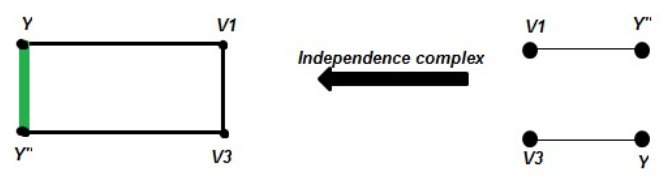

Figure 18: $y$ and $y^{\prime}$ is a dominating set.

Therefore, there is a square we cannot fill.

Then we proved that: If

$$
\psi_{0}(G) \leq 2
$$

then

$$
\eta(\mathscr{I}(G)) \leq 2 .
$$

\section{References}

[1] R. Aharoni, E. Berger, R. Ziv, Independent systems of representatives in weighted graphs, Combinatorica, 27 (2007), 253-267.

[2] K. Kawamura, Independence complex of chordal graphs, Discrete Math., 310 (2010), 2204-2211.

[3] E. Berger, Topological Methods in Matching Theory, Faculty Of Princeton University In Candidacy.

[4] G. A. Dirac, On rigid circuit graphs, Math. sem. Univ. Hamburg, 25 (1961), 71-76

[5] D. Kozov, Combinatorial Algebraic Topology. 